\title{
Evasion of inflammasome activation by microbial pathogens
}

\author{
Tyler K. Ulland, ${ }^{1,2}$ Polly J. Ferguson, ${ }^{3}$ and Fayyaz S. Sutterwala ${ }^{1,2,4,5}$ \\ IInflammation Program, Interdisciplinary Program in Molecular and Cellular Biology, ${ }^{3}$ Department of Pediatrics, and ${ }^{4}$ Department of Internal Medicine, University of lowa, lowa City, lowa, USA \\ ${ }^{5}$ Veterans Affairs Medical Center, lowa City, lowa, USA.
}

\begin{abstract}
Activation of the inflammasome occurs in response to infection with a wide array of pathogenic microbes. The inflammasome serves as a platform to activate caspase-1, which results in the subsequent processing and secretion of the proinflammatory cytokines IL-1 $\beta$ and IL-18 and the initiation of an inflammatory cell death pathway termed pyroptosis. Effective inflammasome activation is essential in controlling pathogen replication as well as initiating adaptive immune responses against the offending pathogens. However, a number of pathogens have developed strategies to evade inflammasome activation. In this Review, we discuss these pathogen evasion strategies as well as the potential infectious complications of therapeutic blockade of IL-1 pathways.
\end{abstract}

\section{Introduction}

The innate immune system plays a critical role in host defense against invading pathogens through the activation of pattern recognition receptors (PRRs) by highly conserved pathogen-associated molecular patterns (PAMPs) or host-derived danger-associated molecular patterns (DAMPs). PRRs include TLR, RIG-I-like receptors (RLR), C-type lectin receptors (CLR), nucleotide-binding domain leucinerich repeat-containing family (NLR), and those belonging to the Pyrin and HIN200 domain-containing (PYHIN) family.

The human NLR family comprises over 23 structurally related proteins, the functions of many of which remain unknown (1). A number of NLRs and the PYHIN family member AIM2 form multiprotein complexes called inflammasomes, which play key roles in regulating both innate and adaptive immune responses. The assembly of an inflammasome results in a platform consisting of an NLR or AIM2, in most cases, the adaptor protein apoptosis-associated speck-like protein containing a caspase activation and recruitment domain (CARD), known as ASC, and the cysteine protease caspase-1 (Figure 1 and ref. 1). Inflammasome activation results in the release of potent proinflammatory mediators and thus is a tightly regulated process, as their inadvertent release could cause collateral tissue damage. Inflammasome activation is generally a two-step process. The priming step results in the transcription of pro-IL-1 $\beta$, pro-IL-18, and certain inflammasome components (2). The second signal, which can be initiated by a variety of stimuli, results in the activation of the inflammasome (2). The two-step process for inflammasome activation is clearly required for NLRP3 inflammasome activation; however, the requirement for a separate priming step is less clear for NLRP1, NLRC4, and AIM2 inflammasomes. Once activated, the inflammasome complex serves as a platform for the autocatalytic cleavage of pro-caspase- 1 into its

Conflict of interest: The authors have declared that no conflict of interest exists. Reference information: J Clin Invest. 2015;125(2):469-477. doi:10.1172/JCI75254. mature activated form. Caspase- 1 in turn cleaves pro-IL-1 $\beta$ and pro-IL-18 into their mature secreted forms. Caspase- 1 activation is also required for the initiation of an inflammatory programmed cell death pathway termed pyroptosis. In addition, inflammasome activation is associated with the rapid release of eicosanoids that drive further inflammation and vascular permeability (3).

NLRP1, NLRP3, NLRC4, and AIM2 are the best-characterized sensors capable of forming inflammasome complexes (Figure 1). Recently, NLRP2, NLRP6, NLRP7, RIG-I, pyrin, and IFI16 have been implicated in the formation of unique inflammasome complexes (4-8); however, additional investigation will be required to establish their precise roles in inflammasome formation and activation. Pyrin, mutations in which cause the autoinflammatory disease familial Mediterranean fever, can also form a caspase-1-activating inflammasome in concert with ASC $(9,10)$. Interestingly, a recent study demonstrated that the Pyrin inflammasome is activated through the sensing of bacterial modification and inactivation of Rho GTPases (11).

Noncanonical inflammasome activation promotes activation of caspase-11, which is important for caspase- 1 activation, IL- $1 \beta$ secretion, and pyroptotic cell death in response to Escherichia coli, Citrobacter rodentium, and Vibrio cholerae (12). Activation of caspase-11 is triggered by the detection of cytosolic acylated lipid A, which is a component of LPS that is present in many Gram-negative bacteria. Of note, intracellular LPS or acylated lipid A is capable of activating caspase-11 independently of TLR4; however, the identity of the receptor that recognizes cytosolic LPS remains unclear $(13,14)$. A recent study demonstrated that human caspase- 4 and caspase- 5 and mouse caspase- 11 were capable of directly binding to LPS and lipid A, resulting in their activation and the initiation of cell death (15).

\section{Pathogen-mediated inflammasome activation}

A single NLRP1 gene is present in humans; in contrast, mice possess three NLRP1 orthologs, Nlrp1a, Nlrp1b, and Nlrp1c. An important structural difference between human NLRP1 and its murine orthologs is that mice lack the N-terminal Pyrin domain (PYD) that 


\section{A AIM2 inflammasome}

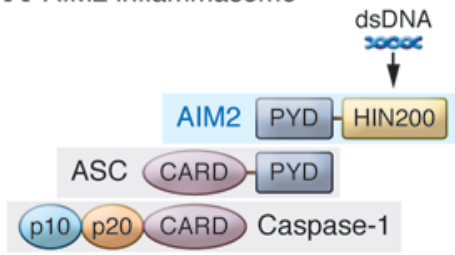

B NLRP1B inflammasome

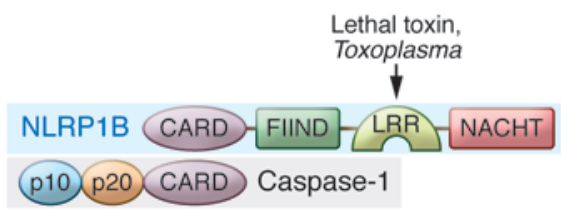

NLRP3 inflammasome

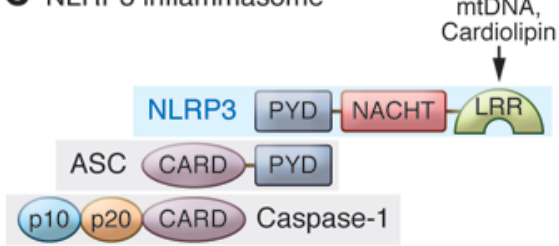

D NLRC4 inflammasome

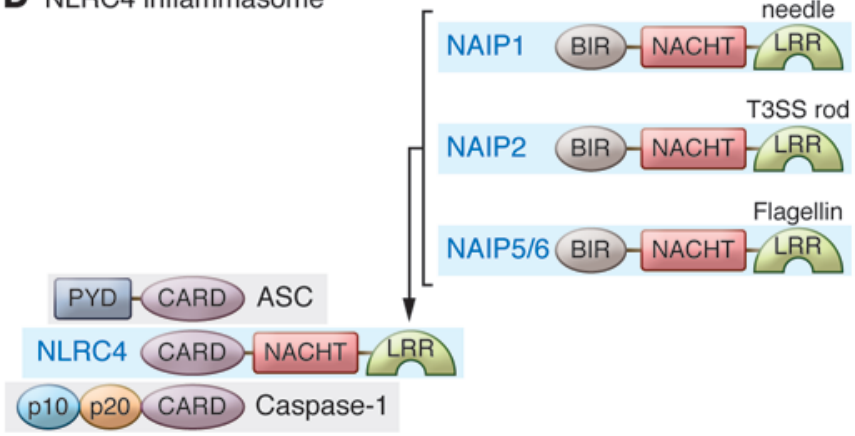

is present in human NLRP1 (1). The murine NLRP1B inflammasome regulates macrophage cell death in response to anthrax lethal toxin (16). NLRP1B also plays an important role in host defense against Bacillus anthracis in vivo (17). Recent studies have demonstrated that the Nlrp1 locus is also required for host defense against the intracellular protozoan parasite Toxoplasma gondii $(18,19)$.

The NLRP3 inflammasome has been associated with numerous pathologic states, including infectious, autoinflammatory, and autoimmune disorders. As such, a wide array of agonists are capable of activating the NLRP3 inflammasome, including those derived from microbes (PAMPs) or from endogenous or environmental sources (DAMPs) (20). Microbial activators of the NLRP3 inflammasome include both Gram-positive and Gram-negative bacteria (Staphylococcus aureus, Listeria monocytogenes, Streptococcus pneumoniae, Neisseria gonorrhoeae, and others) (21), fungi (Candida albicans, Aspergillus fumigatus, Microsporum canis, and others) (22), RNA and DNA viruses (influenza virus, adenovirus, respiratory syncytial virus [RSV], and others) (23), and parasitic pathogens (Plasmodium chabaudi, Leishmania amazonensis, and Schistosoma mansoni) (24). Given the large number of chemically and structurally diverse agonists that are capable of activating the NLRP3 inflammasome, it is unlikely that NLRP3 directly
Figure 1. Schematic of AIM2, NLRP1B, NLRP3, and NLRC4 inflammasomes. (A) The AIM2 inflammasome detects the presence of cytosolic dsDNA via its HIN200 domain. AIM2 then recruits ASC through its N-terminal PYD, which recruits caspase- 1 via its CARD domain. (B) $B$. anthracis lethal toxin and $T$. gondii can induce the activation of the NLRP1B inflammasome. Mouse NLRP1B does not possess a functional N-terminal PYD that is found in human NLRP1; thus, caspase-1 is proposed to interact with its C-terminal CARD. (C) A diverse array of agonists can activate the NLRP3 inflammasome; it is thought that they ultimately lead to mitochondrial dysfunction, resulting in mtDNA and cardiolipin interactions with NLRP3, which leads to its activation. NLRP3 interacts with ASC through an N-terminal PYD, which then recruits caspase-1. (D) NAIP1, NAIP2, and NAIP5/ 6 bind to the T3SS needle and rod proteins and bacterial flagellin, respectively. The NAIP proteins in turn activate the NLRC4 inflammasome. FIIND, domain with function to find; NACHT, nucleotide-binding and oligomerization domain; LRR, leucine-rich repeats; BIR, baculovirus IAP repeat domain; HIN200; HIN2OO domain.

detects the cytosolic presence of these agonists; rather, it probably responds to a cellular stress signal induced by the infectious agents. Recent studies suggest that mitochondrial dysfunction leading to the release of mitochondrial DNA (mtDNA) and the phospholipid cardiolipin triggers activation of the NLRP3 inflammasome $(25,26)$; the current understanding of the mechanism of NLRP3 activation is reviewed in detail elsewhere (20).

The NLRC4 inflammasome is activated by a number of Gram-negative bacteria that possess either a type III (T3SS) or type IV (T4SS) secretion system, including Pseudomonas aeruginosa, Salmonella enterica, Legionella pneumophila, and Shigella flexneri (27). NLRC4 is activated in response to the detection of cytoplasmic flagellin or specific components of the bacterial T3SS or T4SS secretion systems. Activation of the NLRC4 inflammasome requires the involvement of the neuronal apoptosis inhibitor protein (NAIP) subfamily of NLR proteins. Murine NAIP1 binds to the needle protein of the T3SS; NAIP2 recognizes the basal rod structure of the T3SS; NAIP5 and NAIP6 bind to cytosolic flagellin (27). There is only one human NAIP homolog, which binds to the needle protein of the T3SS (27).

The AIM2 inflammasome plays a role in host defense through the recognition of dsDNA within the cytosol. This occurs through direct binding of DNA to the HIN200 domain of AIM2. AIM2 inflammasome activation occurs in response to infection with a number of intracellular bacterial pathogens (Francisella tularensis, Mycobacterium tuberculosis, L. monocytogenes, and others) and viruses (cytomegalovirus, vaccinia virus, and others) (28).

\section{Bacterial effector molecules that facilitate evasion of inflammasome activation}

Given the role of the inflammasome in controlling a wide array of microbial pathogens, it is not surprising that a number of organisms have evolved specific strategies to avoid activation of this innate immune pathway. A number of pathogenic Gram-negative bacteria utilize a T3SS or T4SS to inject effector molecules into the cytoplasmic compartment of the host cell. The T3SS and T4SS are complex macromolecular structures that span both bacterial membranes and include a long, needle-like structure through which the effector molecules pass into the cytoplasm of the eukaryotic host cell. These effector molecules are capable of altering host cell functions, including inflammasome activation. 


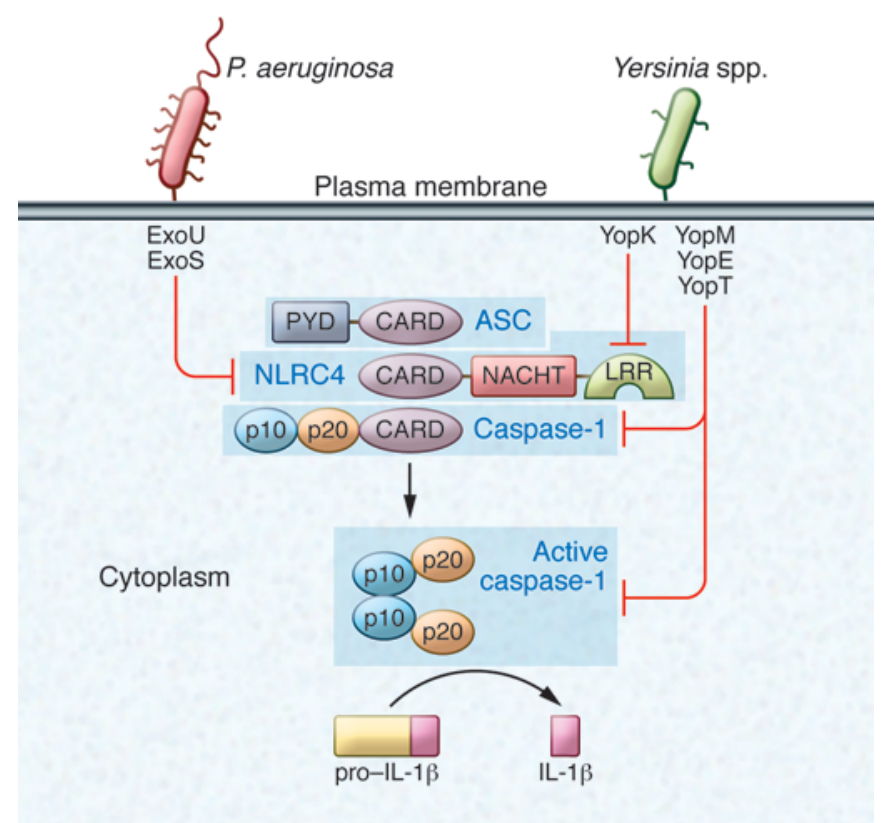

Figure 2. Bacterial effector molecule-mediated inhibition of the NLRC4 inflammasome. Y. pseudotuberculosis YopK is secreted into the host cell through the T3SS; YopK interacts with the translocon structure of the T3SS and effectively masks it from recognition by NAIP/NLRC4. Y. pseudotuberculosis YopM directly inhibits caspase- 1 by binding to its catalytically active site. Y. enterocolitica YopE and YopT inhibit caspase-1 activation through an unknown mechanism. $P$. aeruginosa ExoU and ExoS inhibit NLRC4 inflammasome and caspase-1 activation through an unknown mechanism.

S. enterica serovar typhimurium requires two T3SSs for virulence, and the Salmonella pathogenicity islands SPI-1 and SPI-2 respectively encode these T3SSs. Intracellular S. typhimurium activates both NLRP3 and NLRC4 inflammasomes (29). SPI-1 T3SS-mediated injection of flagellin and the PrgJ rod protein into the macrophage cytoplasm results in NLRC4 inflammasome activation (30-32). During the systemic phase of infection, SPI-1 and flagellin expression are downregulated, allowing $S$. typhimurium to evade NLRC4 inflammasome activation (33). To promote replication within macrophages, S. typhimurium instead relies on the SPI-2-encoded T3SS, whose rod protein, SsaI, is not recognized by the NLRC4 inflammasome (30). S. typhimurium-induced NLRP3 inflammasome activation occurs in a T3SS-independent manner; however, the specific stimuli has yet to be identified (29). S. typhimurium has also developed mechanisms to evade NLRP3 inflammasome activation by utilizing the TCA enzymes aconitase $(\mathrm{acnB})$ and isocitrate dehydrogenase (icdA). Interestingly, $a c n B$ and $i c d A$ mutants induced rapid NLRP3 inflammasome activation through a process that resulted in elevated bacterial citrate and increased mitochondrial reactive oxygen species (34).

In contrast to $S$. typhimurium, Yersinia spp., $P$. aeruginosa, and Vibrio parahaemolyticus have all been shown to utilize secreted effector molecules to subvert inflammasome activation (35-40); we will discuss Yersinia spp. and P. aeruginosa in greater detail below.

$P$. aeruginosa. One of the first reports of inflammasome inhibition by a pathogen described the ability of exoenzyme $\mathrm{U}$ (ExoU) expressing $P$. aeruginosa to inhibit NLRC4 inflammasome activation (Figure 2 and ref. 36). P. aeruginosa has four known effector molecules, ExoS, ExoT, ExoU, and ExoY, which can be secreted into the host cell. ExoU has phospholipase A2 activity, which is required to suppress caspase- 1 activation by an as-yet-unknown mechanism (36).

ExoS, another $P$. aeruginosa effector molecule, is also translocated into host cells through the T3SS (Figure 2). Interestingly, although most $P$. aeruginosa strains carry ExoT and ExoY, the presence of ExoS and ExoU appears to be mutually exclusive (41). ExoS is a Rho GTPase/ADP-ribosyltransferase (ADPR) protein. ExoS-competent bacteria have been shown to inhibit $P$. aeruginosa-induced IL-1 $\beta$ maturation (37). ExoS also appears to effectively switch the mode of death of $P$. aeruginosa-infected cells from proinflammatory caspase-1-dependent pyroptosis to a comparably noninflammatory caspase-3-dependent apoptotic cell death (37). It is not known whether ExoS-induced caspase-3 activation is an active process or a consequence of caspase- 1 inhibition. However, the strategy of inducing cellular death dependent upon caspase-3 may allow the organism to "silently" replicate prior to activation of the innate immune response. The direct link between in vivo virulence and inhibition of inflammasome activation by ExoS- and ExoU-containing $P$. aeruginosa strains is an area that requires further investigation.

Yersinia spp. Pathogens belonging to the genus Yersinia also have effector proteins capable of modulating caspase- 1 activity. A number of species from the genus Yersinia possess the effector molecule YopM that in Y. pseudotuberculosis inhibits caspase-1 activity by binding to the active site of caspase-1 (Figure 2). This was mediated by a four-amino acid sequence in an exposed loop of YopM, which bears similarity to caspase-1 substrate YVAD. The interaction between YopM and caspase- 1 has been proposed to both limit the activity of caspase- 1 and inhibit the assembly of the complete inflammasome complex (39). Consistent with this innate immune evasion function of YopM, Y. pseudotuberculosis strains bearing mutated YopM are highly attenuated in vivo. Further, this attenuation has been demonstrated to be dependent upon caspase-1, thus implicating the ability to subvert the activation of the inflammasome as being an important survival strategy in the pathogenesis of YopM-competent Yersinia (39).

YopE and YopT are Yersinia effector proteins that target the actin cytoskeleton of the host cell and limit phagocytosis of the bacteria through targeting of Rho GTPase family members. YopE and YopT from $Y$. enterocolitica have also been demonstrated to inhibit procaspase-1 oligomerization and maturation when overexpressed (Figure 2). Overexpression of YopE and YopT interferes with Rac-1 activation and significantly inhibits caspase-1 activation (40). This inhibition of caspase- 1 activation by YopE and YopT resulted in diminished macrophage caspase-1-mediated processing of pro-IL-1 $\beta$ into its mature secreted form (40). In a separate study, it was determined that Y. enterocolitica strains with mutant YopE were moderately attenuated in vivo (42). However, Y. enterocolitica YopT mutants did not display any defects in virulence (42). To date, no study has investigated a role for inflammasome activation or IL-1 $\beta$ secretion in the virulence of this mutation in vivo; therefore, additional studies are warranted to determine the role of the inflammasome in response to YopE- or YopT-deficient Yersinia spp.

The final identified mechanism by which Yersinia spp. subverts inflammasome activation is through the activity of YopK (Figure 2). YopK from $Y$. pseudotuberculosis was shown to bind to the T3SS 


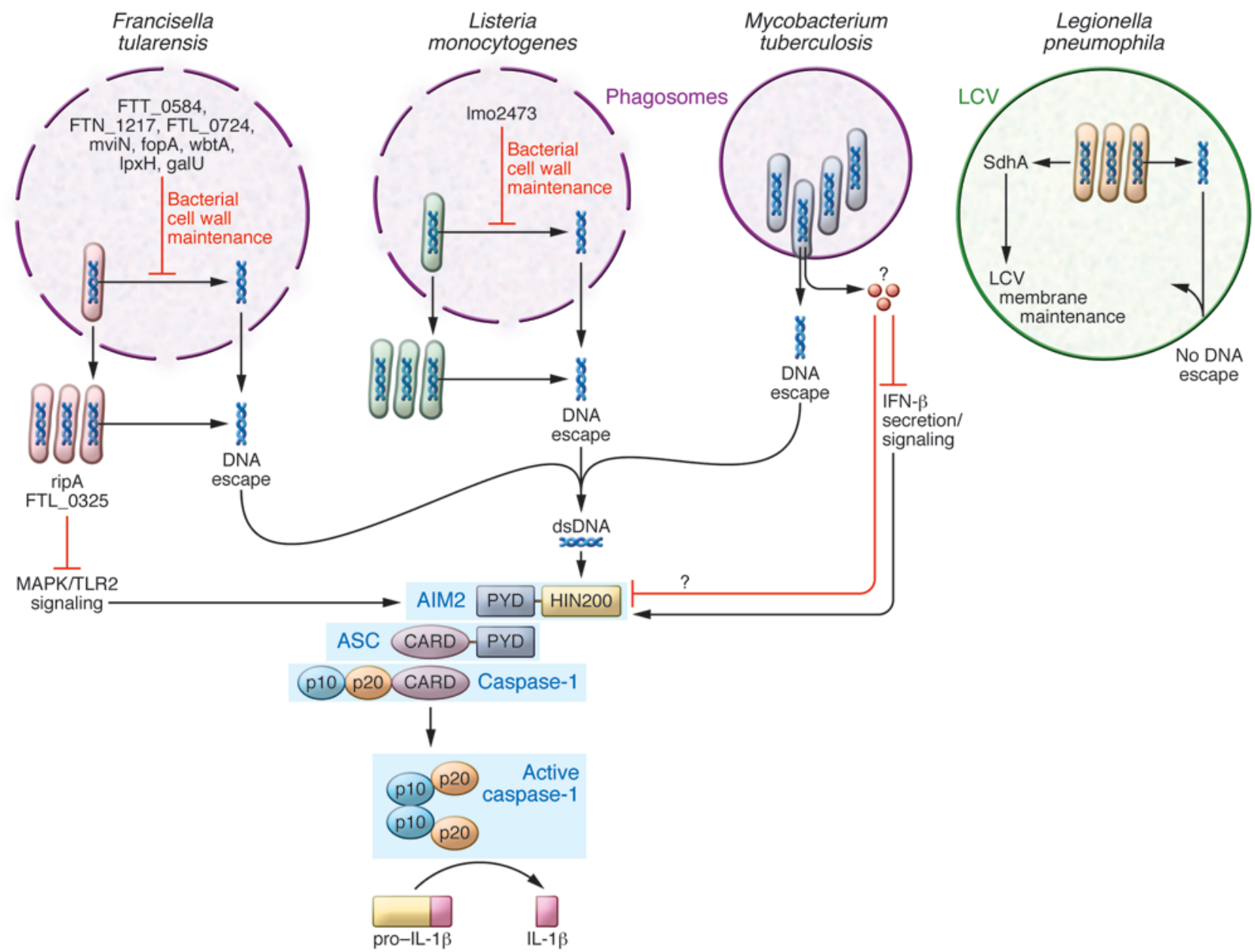

Figure 3. Evasion of AIM2 inflammasome activation by intracellular pathogens. F. tularensis and L. monocytogenes express a number of proteins involved in maintenance of bacterial cell wall integrity. In their absence, increased damage to a subpopulation of bacteria occurs while in the macrophage phagosome, resulting in leakage of bacterial DNA. Once Francisella and Listeria escape into the cytosol of the cell, this DNA is also released into the host cell cytosol, where it interacts with and activates the AIM2 inflammasome. F. tularensis ripA and the gene products of FTL_0325 inhibit MAPK and TLR2 signaling, respectively, and interfere with priming of the AIM2 inflammasome. L. pneumophila secretes the effector molecule into the LCV, where it maintains LCV membrane integrity and prevents Legionella DNA from entering the cytosol and being recognized by AIM2. M. tuberculosis secretes DNA into the cytosol via the ESX-1 secretion system concurrently with an unknown inhibitor of IFN- $\beta$ and also possibly AIM2.

translocon, effectively masking it from recognition by NLRC4 (38). In the absence of YopK, Y. pseudotuberculosis activates caspase-1 in an NLRP3/NLRC4/ASC-dependent manner, resulting in increased bacterial clearance of the YopK mutant in vivo (38).

\section{Preventing DNA release to avoid AIM2 inflammasome activation}

F. tularensis, L. monocytogenes, and L. pneumophila all express proteins that allow the bacteria to evade robust activation of the AIM2 inflammasome. L. monocytogenes is a Gram-positive facultative intracellular pathogen that rapidly replicates within host cells. Following internalization, L. monocytogenes escapes the phagosome and enters the host cell cytosol by a mechanism that is specialized in its ability to prevent the associated induction of host cell death. Mutation of $l m o 2473$, a gene that encodes a protein of unknown function, resulted in L. monocytogenes that hyperactivated the AIM2 inflammasome independently of both NLRC4 and NLRP3 (Figure 3 and ref. 43). This increased AIM2 inflammasome activ- ity was associated with increased IL-1 secretion and pyroptotic cell death. Hyperactivation of the AIM2 inflammasome was linked to impaired cell wall integrity of the lmo2473 mutant, driving an increase in its intracellular lysis. Enhanced bacterial lysis resulted in an increase in DNA release into the cytosol that in turn triggered augmented AIM2 inflammasome activation (43). Therefore, the maintenance of normal bacterial cell wall integrity while in the host cell cytosol, dependent in part upon expression of lmo2473, allowed the bacteria to subvert robust AIM2 activation.

F. tularensis is a virulent Gram-negative bacterium that, after its phagocytosis by the macrophage, escapes the phagosome into the cytosol where it replicates. Although this escape from the phagosome is critical to the survival of $F$.tularensis, it is thought to be associated with damage to a small population of bacteria. As in the case of L. monocytogenes lysis described above, damage of $F$. tularensis is associated with release of dsDNA, resulting in the activation of the AIM2 inflammasome. AIM2 inflammasome activation in turn leads to the secretion of IL-1 $\beta$ and IL-18 and 
the induction of macrophage pyroptosis $(44,45)$. Mutation of the F. tularensis live vaccine strain (LVS) putative lipid II flippase, mviN, resulted in highly attenuated bacteria in in vivo infection models (46). The attenuation of the mviN mutant strain was dependent on the inflammasome, as mice deficient in ASC or caspase-1, but not wild-type mice, succumbed to infection with the mviN mutant strain. The mviN mutant strain also hyperactivates the AIM2 inflammasome in vitro (Figure 3). In addition to $m v i N$, a number of additional $F$. tularensis LVS and F. novicida mutant strains have been identified that also result in increased macrophage cytotoxicity and elevated IL-1 $\beta$ secretion (Figure 3 and refs. 47-50). Importantly, Peng and colleagues demonstrated that these mutations generally result in defects in membraneassociated proteins or in genes involved in O-antigen or LPS biosynthesis (47). This results in increased intracellular lysis of the mutant bacteria, leading to increased bacterial DNA release into the host cell cytosol, which triggers AIM2 inflammasome activation (47). Therefore, the maintenance of bacterial membrane stability is required as a strategy for F.tularensis to avoid AIM2 inflammasome activation. In addition, mutations in ripA and FTL_O325 in F. tularensis LVS resulted in hypercytotoxicity and increased IL- $1 \beta$ secretion. These gene products were not involved in maintaining bacterial cell wall integrity, but instead interfered with MAPK- and TLR2-signaling pathways and hence interfered with the priming step required for AIM2 inflammasome activation $(48,51)$.

L. pneumophila is a Gram-negative intracellular bacterium that activates the NLRC4 inflammasome via its Dot/Icm T4SS. L. pneumophila resides within a structure called a Legionellacontaining vacuole (LCV) that avoids fusion with lysosomes, thereby maintaining a replicative niche for this pathogen within the macrophage. The Dot/Icm-translocated effector molecule SdhA is required for L. pneumophila intracellular growth. Mutant bacteria deficient in SdhA induced elevated IL-1 $\beta$ secretion and macrophage pyroptosis that was dependent on AIM2 inflammasome activation, but independent of the flagellin-sensing NLRC4 inflammasome pathway (52). Interestingly, SdhA was required to maintain LCV membrane integrity, and thus its absence drove Legionella DNA release into the cytosol and increased AIM2 but not NLRC4 inflammasome activation (52).

M. tuberculosis possesses an ESX-1 secretion system through which bacterial DNA gains entry into the host cell cytosol. Interestingly, the DNA that enters the host cell does not result in AIM2 inflammasome activation. ESX-1-competent M. tuberculosis, but not other closely related species of Mycobacterium, inhibits secretion of IFN- $\beta$ by host cells as well as IFN- $\beta$-mediated signaling (53). This $M$. tuberculosis-mediated reduction in IFN- $\beta$ secretion was partially responsible for the ability of $M$. tuberculosis to inhibit AIM2 inflammasome activation, as type I IFN signaling is required for AIM2 inflammasome activation (54). Hence, the ESX-1-dependent cosecretion into the host cell cytosol of a putative IFN- $\beta$ inhibitor (and/or AIM2 inhibitor) along with M. tuberculosis DNA may allow M. tuberculosis to evade AIM2 inflammasome activation. The identity of the M. tuberculosisderived inhibitor of IFN- $\beta$ and/or AIM2 remains unknown. The relevance of these findings to in vivo host defense against M. tuberculosis also remains to be addressed.

\section{Evasion of inflammasome activation by pathogen decoy proteins}

An inflammasome evasion strategy that has been described in viruses is the expression of viral decoy proteins that attenuate inflammasome activation. Kaposi's sarcoma-associated herpes virus (KSHV) encodes an NLRP1 homolog that lacks PYD and CARD, interacts with host NLRP1, NLRP3, and NOD2, and inhibits virally induced IL-1 $\beta$ secretion. The authors suggest that this ability of KSHV to inhibit inflammasome activation may contribute to the establishment of long-term viral persistence (55).

Johnston et al. identified a poxvirus-encoded PYD-containing protein, M13L, which interacted with ASC and inhibited subsequent inflammasome activation. Deletion of $M 13 L$ resulted in attenuation of myxoma virus in rabbits in vivo (56). Cowpox virus and other orthopox viruses encode a protein that can also inhibit caspase-1 activity (57). The cowpox-encoded cytokine response modifier A (CrmA) protein serves as a pseudo-target for active caspase- 1 that, upon cleavage, covalently bonds with a cysteine in the active site of caspase-1, rendering it inactive. This inactivation is very potent, occurs at very low concentrations of CrmA, and is important to virulence, as cowpox viruses lacking CrmA are highly attenuated in vivo (57-59). These observations further illustrate the importance of inflammasome subversion mechanisms as a survival strategy for pathogens.

\section{Safety of IL-1 inhibitors}

The clinical use of biologic agents to modulate specific inflammatory pathways has grown exponentially in the past decade. Given the clear overlap in the pathways that are critical in the control of microbial pathogens and those that drive pathologic autoimmune and autoinflammatory diseases, it is not surprising that therapeutic blockade of cytokines for the treatment of autoimmune and autoinflammatory diseases could result in severe infectious complications. This is exemplified by our experience with TNF- $\alpha$ antagonists, including etanercept, adalimumab, and infliximab. Postmarketing data revealed a dramatic increase in the number of cases of M. tuberculosis reactivation related to anti-TNF- $\alpha$ treatment $(60,61)$. Given that inflammasome pathways play such a critical role in the control of numerous pathogens and that a number of pathogens themselves have developed strategies to specifically evade this innate immune pathway, it seems likely that blockade of IL-1 $1 \beta$ would be accompanied by a significantly increased risk of both serious and opportunistic infections. Surprisingly, to date, antagonists of IL-1 have been shown to have an excellent safety profile that is better than nearly all other widely used biologics that inhibit inflammatory cytokines. The reason for the remarkable safety profiles of anakinra, rilonacept, canakinumab, and gevokizumab is not at all clear but will be considered below.

Anakinra. The majority of the safety data on inhibiting the IL-1 pathway comes from studies using anakinra, the first IL-1 inhibitor available on the market. Anakinra is a recombinant human IL-1 receptor antagonist (rIL-1Ra) that mimics the action of the natural antagonist IL-1Ra.

The earliest large-scale trials utilizing anakinra were performed in the 1990s in sepsis, where it was added to standard therapy in an attempt to improve outcomes and to decrease multiorgan system failure (62-64). The striking feature of these trials 
was that the treatment of over 1,000 actively septic patients with anakinra (intravenously and in high doses) resulted in no serious safety concerns (62-64). However, there was not a significant therapeutic benefit in sepsis (65).

The therapeutic benefit of anakinra use was more apparent in the treatment of rheumatoid arthritis (RA); anakinra was FDA approved for this indication in 2001. Campion et al. reported preliminary safety data in 175 patients with active RA treated with anakinra at varying doses (66). The trial lasted 7 weeks; the most common adverse event was injection-site reactions, causing $5 \%$ of the patients to withdraw from the study, followed by gastrointestinal symptoms. Sixteen percent had an infectious event. There was no placebo arm (66). Subsequent double-blind, randomized, placebo-controlled multicenter trials were performed; over 2,000 patients with RA were enrolled and treated with anakinra, with the study confirming its excellent safety profile (67, 68). There was no significant increase in serious infections (6771); however, Fleischmann et al. studied 1,346 patients with RA who received anakinra in a 30-month open-label extension and reported an increase in exposure-adjusted event rate for serious infections in the anakinra-treated group (5.37 events/100 patient years) versus controls (1.65 events/100 patient years). However, much of the increased risk of serious infection in this open-label extension was attributed to concurrent corticosteroid use (72). Despite this very favorable safety profile when used as the only biologic, the safety profile is unfavorable with combination biologic therapy. Anakinra in combination with the TNF inhibitor etanercept resulted in more frequent and more severe infections with no added efficacy above etanercept alone (73). Therefore, the use of anakinra with another biologic cytokine-blocking agent should be avoided if at all possible.

Anakinra was subsequently approved by the FDA for use in neonatal onset multisystem inflammatory disorder (NOMID), which is a severe form of cryopyrin-associated periodic syndromes (CAPS) (74). Anakinra has been utilized in a number of other conditions, including other monogenic autoinflammatory disorders (75), such as systemic-onset juvenile idiopathic arthritis $(76,77)$, adult-onset Still disease (AOSD) $(78,79)$, gout $(80)$, polyarticular juvenile idiopathic arthritis (sJIA) (81), diabetes (82), heart disease (83-85), and chronic granulomatous disease (86), among many others (87). In these disorders, anakinra was well tolerated except for injectionsite reactions and a slight increase in nonserious viral respiratory infections. To date, the development of opportunistic infections in individuals taking anakinra has been exceedingly rare (87).

Rilonacept. Rilonacept is a dimeric fusion protein consisting of the ligand-binding domains of the human IL-1R extracellular domains (IL-1RI and IL-1 receptor accessory protein) linked to the Fc portion of human IgG1. It acts as a soluble decoy receptor by binding to IL-1 $\beta$ and preventing its interaction with IL-1R on the cell surface. In 2008, the FDA approved rilonacept for certain forms of CAPS, granting it orphan drug status.

Goldbach-Mansky et al. reported the result of a small openlabel trial of rilonacept in 5 CAPS patients that showed clinical improvement; they did not observe any drug-related adverse events (88). That same year, Hoffman et al. published results on the efficacy and safety of rilonacept in 47 patients with CAPS (89). Rilonacept was generally well tolerated, with injection-site reac- tions and nonserious viral upper respiratory infections being the most common adverse events $(89,90)$. There were 2 deaths during the study: a 71-year-old female who died after developing sinusitis and pneumococcal meningitis and a 37-year-old who died of a myocardial infarction. Subsequently, studies of rilonacept in other autoinflammatory disorders including gout $(91,92)$, sJIA $(93,94)$, AOSD (95), familial Mediterranean fever (96), and Schnitzler syndrome have been reported (97). Again, blockade of IL-1 with this longer-acting agent was well tolerated, with no increased risk of serious or opportunistic infections.

Canakinumab and gevokizumab. There are several anti-IL-1 $\beta$ monoclonal antibodies available, including canakinumab and gevokizumab $(98,99)$. Canakinumab is a fully humanized IgG1 anti-IL-1 $\beta$ monoclonal antibody that binds to human IL-1 $\beta$ with high specificity and neutralizes the bioactivity of this cytokine. These agents have been utilized to treat a number of inflammatory disorders, including CAPS $(98,100,101)$, other monogenic autoinflammatory disorders (75), gout (102), type 1 diabetes (103), type 2 diabetes $(104,105)$, RA (106), sJIA (107), Schnitzler syndrome (108), and Behçet syndrome (109) as well as other inflammatory conditions (87).

Gevokizumab is a monoclonal anti-IL-1 $\beta$ antibody that negatively modulates IL- $1 \beta$ signaling through an allosteric mechanism. It decreases the binding affinity of IL- $1 \beta$ for the IL- 1 receptor type I (IL-1RI) signaling receptor, but not the IL-1 counterregulatory decoy receptor (IL-1 receptor type II) (110). It does not interfere with IL-1Ra or block IL-1 $\beta$ binding to the soluble forms of the IL-1 receptors (111). Gevokizumab inhibits both the binding of IL-1 $\beta$ to IL-1RI and the subsequent recruitment of IL-1 accessory protein, primarily by reducing the association rates of these interactions; as such, it is a unique inhibitor of IL-1 $\beta$ signaling (110). Initial studies with this monoclonal antibody have been in the treatment of type 2 diabetes $(99,112)$ and Behçet disease (113).

The safety profile of the longer-acting IL-1-blocking agents resembles that of anakinra. However, these agents are newer and a smaller number of patients have been treated; therefore, the long-term safety profile has yet to be fully delineated.

\section{Concluding remarks}

The inflammasome is a critical mechanism by which the innate immune system recognizes and limits pathogenic insults. The inflammasome signals and coordinates the response of a number of different cell types primarily through the cytokines IL-1 $\beta$ and IL-18 and through the initiation of pyroptotic cell death. It is evident that the modulation of inflammasome activity is an important strategy employed by a number of pathogens to subvert the normal innate immune response. With the recent introduction of a number of different therapies targeting IL-1 $\beta$ and its receptor, it has become increasingly important to understand the interplay of pathogens and host in the context of inflammasome activation. Surprisingly, the clinical use of inhibitors of IL-1, such as anakinra, rilonacept, canakinumab, and gevokizumab, has been associated with exceedingly few reported infectious complications. Inflammasome-driven pyroptosis and IL-18 production play nonredundant roles in host defense against pathogens that are not blocked by the inhibition of IL-1 signaling. It may be that the unexpected safety profile for IL-1 
blockade is due to this narrow function. As additional therapeutic strategies to inhibit this pathway emerge, careful attention will be required to determine whether inhibition of inflammasomes in toto is similarly seemingly well tolerated. In addition, as the use of longer-acting IL-1 inhibitors increases, patterns of infectious complications may declare themselves in select patient populations. Finally, as new therapies are developed to treat infectious disease, especially therapies against pathogens that subvert inflammasome activation, it may prove beneficial to investigate the use of adjunctive treatments that trigger inflammasome activation to enhance the innate immune responses against invading pathogens.

\section{Acknowledgments}

We thank Suzanne Cassel for helpful discussion and critical review of this manuscript. NIH grants R01 AR059703 (to P.J. Ferguson) and R01 AI087630 (to F.S. Sutterwala) supported this work. The Inflammation Program (to T.K. Ulland and F.S. Sutterwala) is supported by resources and use of facilities at the Veterans Affairs Medical Center, Iowa City, Iowa, USA.

Address correspondence to: Fayyaz Sutterwala, University of Iowa Carver College of Medicine, 2501 Crosspark Road, D156 MTF, Coralville, Iowa 52241, USA. Phone: 319.335.4261; E-mail: fayyaz-sutterwala@uiowa.edu.
1. Schroder K, Tschopp J. The inflammasomes. Cell. 2010;140(6):821-832.

2. Lamkanfi M, Dixit VM. Mechanisms and functions of inflammasomes. Cell. 2014;157(5):1013-1022.

3. von Moltke J, et al. Rapid induction of inflammatory lipid mediators by the inflammasome in vivo. Nature. 2012;490(7418):107-111.

4. Elinav E, et al. NLRP6 inflammasome regulates colonic microbial ecology and risk for colitis. Cell. 2011;145(5):745-757.

5. Khare S, et al. An NLRP7-containing inflammasome mediates recognition of microbial lipopeptides in human macrophages. Immunity. 2012;36(3):464-476.

6. Poeck H, et al. Recognition of RNA virus by RIG-I results in activation of CARD9 and inflammasome signaling for interleukin $1 \beta$ production. Nat Immunol. 2010;11(1):63-69.

7. Kerur N, et al. IFI16 acts as a nuclear pathogen sensor to induce the inflammasome in response to Kaposi Sarcoma-associated herpesvirus infection. Cell Host Microbe. 2011;9(5):363-375.

8. Chae JJ, et al. Gain-of-function Pyrin mutations induce NLRP3 protein-independent interleukin$1 \beta$ activation and severe autoinflammation in mice. Immunity. 2011;34(5):755-768.

9. Seshadri S, Duncan MD, Hart JM, Gavrilin MA, Wewers MD. Pyrin levels in human monocytes and monocyte-derived macrophages regulate IL-1 $\beta$ processing and release. J Immunol. 2007;179(2):1274-1281.

10. Yu JW, et al. Cryopyrin and pyrin activate caspase-1, but not NF- $\mathrm{\kappa B}$, via ASC oligomerization. Cell Death Differ. 2006;13(2):236-249.

11. $\mathrm{Xu} \mathrm{H}$, et al. Innate immune sensing of bacterial modifications of Rho GTPases by the Pyrin inflammasome. Nature. 2014;513(7517):237-241.

12. Kayagaki N, et al. Non-canonical inflammasome activation targets caspase-11. Nature. 2011;479(7371):117-121.

13. Kayagaki $\mathrm{N}$, et al. Noncanonical inflammasome activation by intracellular LPS independent of TLR4. Science. 2013;341(6151):1246-1249.

14. Hagar JA, Powell DA, Aachoui Y, Ernst RK, Miao EA. Cytoplasmic LPS activates caspase-11: implications in TLR4-independent endotoxic shock. Science. 2013;341(6151):1250-1253.

15. Shi J, et al. Inflammatory caspases are innate immune receptors for intracellular LPS. Nature. 2014;514(7521):187-192.

16. Boyden ED, Dietrich WF. Nalp1b controls mouse macrophage susceptibility to anthrax lethal toxin. Nat Genet. 2006;38(2):240-244.

17. Terra JK, et al. Cutting edge: resistance to Bacillus anthracis infection mediated by a lethal toxin sensitive allele of Nalp1b/Nlrp1b. J Immunol. 2010;184(1):17-20.

18. Ewald SE, Chavarria-Smith J, Boothroyd JC. NLRP1 is an inflammasome sensor for Toxoplasma gondii. Infect Immun. 2014;82(1):460-468.

19. Gorfu G, et al. Dual role for inflammasome sensors NLRP1 and NLRP3 in murine resistance to Toxoplasma gondii. MBio. 2014;5(1):e01117-13.

20. Sutterwala FS, Haasken S, Cassel SL. Mechanism of NLRP3 inflammasome activation. Ann N Y Acad Sci. 2014;1319(1):82-95.

21. Vladimer GI, Marty-Roix R, Ghosh S, Weng D, Lien E. Inflammasomes and host defenses against bacterial infections. Curr Opin Microbiol. 2013;16(1):23-31.

22. Joly S, Sutterwala FS. Fungal pathogen recognition by the NLRP3 inflammasome. Virulence. 2010;1(4):276-280.

23. Lupfer C, Kanneganti TD. The expanding role of NLRs in antiviral immunity. Immunol Rev. 2013;255(1):13-24.

24. Clay GM, Sutterwala FS, Wilson ME. NLR proteins parasitic disease. Immunol Res. 2014;59(1-3):142-152.

25. Nakahira K, et al. Autophagy proteins regulate innate immune responses by inhibiting the release of mitochondrial DNA mediated by the NALP3 inflammasome. Nat Immunol. 2011;12(3):222-230.

26. Iyer SS, et al. Mitochondrial cardiolipin is required for Nlrp3 inflammasome activation. Immunity. 2013;39(2):311-323.

27. Gong YN, Shao F. Sensing bacterial infections by NAIP receptors in NLRC4 inflammasome activation. Protein Cell. 2012;3(2):98-105.

28. Schattgen SA, Fitzgerald KA. The PYHIN protein family as mediators of host defenses. Immunol Rev. 2011;243(1):109-118.

29. Broz P, Newton K, Lamkanfi M, Mariathasan S, Dixit VM, Monack DM. Redundant roles for inflammasome receptors NLRP3 and NLRC4 in host defense against Salmonella. J Exp Med. 2010;207(8):1745-1755.

30. Miao EA, et al. Innate immune detection of the type III secretion apparatus through the NLRC4 inflammasome. Proc Natl Acad Sci U S A. 2010;107(7):3076-3080.

31. Miao EA, et al. Cytoplasmic flagellin activates caspase- 1 and secretion of interleukin $1 \beta$ via Ipaf.
Nat Immunol. 2006;7(6):569-575.

32. Franchi L, et al. Cytosolic flagellin requires Ipaf for activation of caspase- 1 and interleukin $1 \beta$ in salmonella-infected macrophages. Nat Immunol. 2006; 7(6):576-582.

33. Ibarra JA, Steele-Mortimer O. Salmonella - the ultimate insider. Salmonella virulence factors that modulate intracellular survival. Cell Microbiol. 2009;11(11):1579-1586.

34. Wynosky-Dolfi MA, et al. Oxidative metabolism enables Salmonella evasion of the NLRP3 inflammasome. J Exp Med. 2014;211(4):653-668.

35. Higa N, et al. Vibrio parahaemolyticus effector proteins suppress inflammasome activation by interfering with host autophagy signaling. PLoS Pathog. 2013;9(1):e1003142.

36. Sutterwala FS, Mijares LA, Li L, Ogura Y, Kazmierczak BI, Flavell RA. Immune recognition of Pseudomonas aeruginosa mediated by the IPAF/NLRC4 inflammasome. J Exp Med. 2007;204(13):3235-3245.

37. Galle $\mathrm{M}$, et al. The Pseudomonas aeruginosa Type III secretion system plays a dual role in the regulation of caspase- 1 mediated IL-1beta maturation. J Cell Mol Med. 2008;12(5A):1767-1776.

38. Brodsky IE, et al. A Yersinia effector protein promotes virulence by preventing inflammasome recognition of the type III secretion system. Cell Host Microbe. 2010;7(5):376-387.

39. LaRock CN, Cookson BT. The Yersinia virulence effector YopM binds caspase-1 to arrest inflammasome assembly and processing. Cell Host Microbe. 2012;12(6):799-805.

40. Schotte P, Denecker G, Van Den Broeke A, Vandenabeele P, Cornelis GR, Beyaert R. Targeting Rac1 by the Yersinia effector protein YopE inhibits caspase1-mediated maturation and release of interleukin13. JBiol Chem. 2004;279(24):25134-25142.

41. Feltman H, Schulert G, Khan S, Jain M, Peterson L, Hauser AR. Prevalence of type III secretion genes in clinical and environmental isolates of Pseudomonas aeruginosa. Microbiology. 2001;147(pt 10):2659-2669.

42. Trulzsch K, Sporleder T, Igwe EI, Russmann H, Heesemann J. Contribution of the major secreted yops of Yersinia enterocolitica O:8 to pathogenicity in the mouse infection model. Infect Immun. 2004;72(9):5227-5234.

43. Sauer JD, Witte CE, Zemansky J, Hanson B, Lauer $\mathrm{P}$, Portnoy DA. Listeria monocytogenes triggers AIM2-mediated pyroptosis upon infrequent bacteriolysis in the macrophage cytosol. Cell Host 
Microbe. 2010;7(5):412-419.

44. Fernandes-Alnemri T, et al. The AIM2 inflammasome is critical for innate immunity to Francisella tularensis. Nat Immunol. 2010;11(5):385-393.

45. Jones JW, et al. Absent in melanoma 2 is required for innate immune recognition of Francisella tularensis. Proc Natl Acad Sci U S A. 2010;107(21):9771-9776.

46. Ulland TK, et al. Cutting edge: mutation of Francisella tularensis mviN leads to increased macrophage absent in melanoma 2 inflammasome activation and a loss of virulence. J Immunol. 2010;185(5):2670-2674.

47. Peng K, Broz P, Jones J, Joubert LM, Monack D. Elevated AIM2-mediated pyroptosis triggered by hypercytotoxic Francisella mutant strains is attributed to increased intracellular bacteriolysis. Cell Microbiol. 2011;13(10):1586-1600.

48. Huang MT, et al. Deletion of ripA alleviates suppression of the inflammasome and MAPK by Francisella tularensis. J Immunol. 2010;185(9):5476-5485.

49. Jayakar HR, et al. A galU mutant of Francisella tularensis is attenuated for virulence in a murine pulmonary model of tularemia. BMC Microbiol. 2011;11:179.

50. Weiss DS, Brotcke A, Henry T, Margolis JJ, Chan K, Monack DM. In vivo negative selection screen identifies genes required for Francisella virulence. Proc Natl Acad Sci US A. 2007;104(14):6037-6042.

51. Dotson RJ, et al. Repression of inflammasome by Francisella tularensis during early stages of infection. J Biol Chem. 2013;288(33):23844-23857.

52. Ge J, Gong YN, Xu Y, Shao F. Preventing bacterial DNA release and absent in melanoma 2 inflammasome activation by a Legionella effector functioning in membrane trafficking. Proc Natl Acad Sci U S A. 2012;109(16):6193-6198.

53. Shah S, et al. Cutting edge: Mycobacterium tuberculosis but not nonvirulent mycobacteria inhibits IFN- $\beta$ and AIM 2 inflammasome-dependent IL- $1 \beta$ production via its ESX-1 secretion system. Jimmunol. 2013;191(7):3514-3518.

54. Henry T, Brotcke A, Weiss DS, Thompson LJ, Monack DM. Type I interferon signaling is required for activation of the inflammasome during Francisella infection. J Exp Med. 2007;204(5):987-994.

55. Gregory SM, et al. Discovery of a viral NLR homolog that inhibits the inflammasome. Science. 2011;331(6015):330-334

56. Johnston JB, et al. A poxvirus-encoded pyrin domain protein interacts with ASC-1 to inhibit host inflammatory and apoptotic responses to infection. Immunity. 2005;23(6):587-598.

57. Best SM. Viral subversion of apoptotic enzymes: escape from death row. Annu Rev Microbiol. 2008;62:171-192.

58. Ray CA, et al. Viral inhibition of inflammation: cowpox virus encodes an inhibitor of the interleukin-1 $\beta$ converting enzyme. Cell. 1992;69(4):597-604.

59. Swanson R, Raghavendra MP, Zhang W, Froelich C, Gettins PG, Olson ST. Serine and cysteine proteases are translocated to similar extents upon formation of covalent complexes with serpins. Fluorescence perturbation and fluorescence resonance energy transfer mapping of the protease binding site in CrmA complexes with granzyme B and caspase-1. J Biol Chem. 2007;282(4):2305-2313.

60. Fellermann K. Adverse events of tumor necrosis factor inhibitors. Dig Dis. 2013;31(3-4):374-378.

61. Keane J, et al. Tuberculosis associated with infliximab, a tumor necrosis factor alpha-neutralizing agent. N Engl J Med. 2001;345(15):1098-1104.

62. Opal SM, et al. Confirmatory interleukin-1 receptor antagonist trial in severe sepsis: a phase III, randomized, double-blind, placebo-controlled, multicenter trial. The Interleukin-1 Receptor Antagonist Sepsis Investigator Group. Crit Care Med.1997;25(7):1115-1124.

63. Fisher CJ Jr, et al. Recombinant human interleukin 1 receptor antagonist in the treatment of patients with sepsis syndrome. Results from a randomized, double-blind, placebo-controlled trial. Phase III rhIL-1ra Sepsis Syndrome Study Group. JAMA. 1994;271(23):1836-1843.

64. Fisher CJ Jr, et al. Initial evaluation of human recombinant interleukin-1 receptor antagonist in the treatment of sepsis syndrome: a randomized open-label, placebo-controlled multicenter trial. Crit Care Med.1994;22(1):12-21.

65. Marshall JC. Such stuff as dreams are made on: mediator-directed therapy in sepsis. Nat Rev Drug Discov. 2003;2(5):391-405.

66. Campion GV, Lebsack ME, Lookabaugh J, Gordon G, Catalano M. Dose-range and dosefrequency study of recombinant human interleukin-1 receptor antagonist in patients with rheumatoid arthritis. The IL-1Ra Arthritis Study Group. Arthritis Rheum. 1996;39(7):1092-1101.

67. Bresnihan B, et al. Treatment of rheumatoid arthritis with recombinant human interleukin-1 receptor antagonist. Arthritis Rheum. 1998;41(12):2196-2204.

68. Nuki G, et al. Long-term safety and maintenance of clinical improvement following treatment with anakinra (recombinant human interleukin-1 receptor antagonist) in patients with rheumatoid arthritis: extension phase of a randomized, double-blind, placebo-controlled trial. Arthritis Rheum. 2002;46(11):2838-2846.

69. Cohen S, et al. Treatment of rheumatoid arthritis with anakinra, a recombinant human interleukin-1 receptor antagonist, in combination with methotrexate: results of a twenty-fourweek, multicenter, randomized, double-blind, placebo-controlled trial. Arthritis Rheum 2002;46(3):614-624.

70. Fleischmann RM, et al. Anakinra, a recombinant human interleukin-1 receptor antagonist (r-metHuIL-1ra), in patients with rheumatoid arthritis: A large, international, multicenter, placebo-controlled trial. Arthritis Rheum 2003;48(4):927-934.

71. Cohen SB, et al. A multicentre, double blind, randomised, placebo controlled trial of anakinra (Kineret), a recombinant interleukin 1 receptor antagonist, in patients with rheumatoid arthritis treated with background methotrexate. Ann Rheum Dis. 2004;63(9):1062-1068.

72. Fleischmann RM, et al. Safety of extended treatment with anakinra in patients with rheumatoid arthritis. Ann Rheum Dis. 2006;65(8):1006-1012.

73. Genovese MC, et al. Combination therapy with etanercept and anakinra in the treatment of patients with rheumatoid arthritis who have been treated unsuccessfully with methotrexate. Arthritis Rheum. 2004;50(5):1412-1419.

74. Sibley $\mathrm{CH}$, et al. Sustained response and prevention of damage progression in patients with neonatal-onset multisystem inflammatory disease treated with anakinra: a cohort study to determine three- and five-year outcomes. Arthritis Rheum. 2012;64(7):2375-2386.

75. Almeida de Jesus A, Goldbach-Mansky R. Monogenic autoinflammatory diseases: concept and clinical manifestations. Clin Immunol. 2013;147(3):155-174.

76. Pascual V, Allantaz F, Arce E, Punaro M, Banchereau J. Role of interleukin-1 (IL-1) in the pathogenesis of systemic onset juvenile idiopathic arthritis and clinical response to IL-1 blockade. JExp Med. 2005;201(9):1479-1486.

77. Verbsky JW, White AJ. Effective use of the recombinant interleukin 1 receptor antagonist anakinra in therapy resistant systemic onset juvenile rheumatoid arthritis. J Rheumatol. 2004;31(10):2071-2075.

78. Fitzgerald AA, Leclercq SA, Yan A, Homik JE, Dinarello CA. Rapid responses to anakinra in patients with refractory adult-onset Still's disease. Arthritis Rheum. 2005;52(6):1794-1803.

79. Lequerre T, et al. Interleukin-1 receptor antagonist (anakinra) treatment in patients with systemic-onset juvenile idiopathic arthritis or adult onset Still disease: preliminary experience in France. Ann Rheum Dis. 2008;67(3):302-308.

80. So A, De Smedt T, Revaz S, Tschopp J. A pilot study of IL-1 inhibition by anakinra in acute gout. Arthritis Res Ther. 2007;9(2):R28.

81. Ilowite $\mathrm{N}$, et al. Anakinra in the treatment of polyarticular-course juvenile rheumatoid arthritis: safety and preliminary efficacy results of a randomized multicenter study. Clin Rheumatol. 2009;28(2):129-137.

82. Larsen CM, et al. Interleukin-1-receptor antagonist in type 2 diabetes mellitus. $N$ Engl J Med 2007;356(15):1517-1526.

83. Abbate A, et al. Effects of interleukin-1 blockade with anakinra on adverse cardiac remodeling and heart failure after acute myocardial infarction [from the Virginia Commonwealth University-Anakinra Remodeling Trial (2) (VCU-ART2) pilot study]. Am J Cardiol. 2013;111(10):1394-1400.

84. Ikonomidis I, et al. Inhibition of interleukin-1 by anakinra improves vascular and left ventricular function in patients with rheumatoid arthritis. Circulation. 2008;117(20):2662-2669.

85. Van Tassell BW, et al. Effects of interleukinblockade with anakinra on aerobic exercise capacity in patients with heart failure and preserved ejection fraction (from the D-HART pilot study). Am J Cardiol. 2014;113(2):321-327.

86. van de Veerdonk FL, Netea MG, Dinarello CA, van der Meer JW. Anakinra for the inflammatory complications of chronic granulomatous disease. Neth J Med. 2011;69(2):95.

87. Dinarello CA, van der Meer JW. Treating inflammation by blocking interleukin-1 in humans Semin Immunol. 2013;25(6):469-484

88. Goldbach-Mansky R, et al. A pilot study to evaluate the safety and efficacy of the long- 
acting interleukin-1 inhibitor rilonacept (interleukin-1 Trap) in patients with familial cold autoinflammatory syndrome. Arthritis Rheum. 2008;58(8):2432-2442.

89. Hoffman HM, et al. Efficacy and safety of rilonacept (interleukin-1 Trap) in patients with cryopyrin-associated periodic syndromes: results from two sequential placebo-controlled studies. Arthritis Rheum. 2008;58(8):2443-2452.

90. Hoffman HM, et al. Long-term efficacy and safety profile of rilonacept in the treatment of cryopryin-associated periodic syndromes: results of a 72-week open-label extension study. Clin Ther. 2012;34(10):2091-2103.

91. Terkeltaub R, et al. The interleukin 1 inhibitor rilonacept in treatment of chronic gouty arthritis: results of a placebo-controlled, monosequence crossover, non-randomised, single-blind pilot study. Ann Rheum Dis. 2009;68(10):1613-1617.

92. Schumacher HR Jr, et al. Rilonacept (interleukin-1 trap) in the prevention of acute gout flares during initiation of urate-lowering therapy: results of a phase II randomized, double-blind, placebo-controlled trial. Arthritis Rheum. 2012;64(3):876-884.

93. Ilowite NT, for the RAPPORT Investigators. Randomized, double-blind, placebo-controlled trial of the efficacy and safety of rilonacept in the treatment of systemic juvenile idiopathic arthritis [published online ahead of print December 15, 2014]. Arthritis Rheum. doi:10.1002/art.38991.

94. Lovell DJ, et al. Long-term safety and efficacy of rilonacept in patients with systemic juvenile idiopathic arthritis. Arthritis Rheum. 2013;65(9):2486-2496.

95. Petryna O, Cush JJ, Efthimiou P. IL-1 Trap rilonacept in refractory adult onset Still's disease. Ann Rheum Dis. 2012;71(12):2056-2057.

96. Hashkes PJ, et al. Rilonacept for colchicineresistant or -intolerant familial Mediterranean fever: a randomized trial. Ann Intern Med. 2012;157(8):533-541.

97. Krause K, et al. Efficacy and safety of the interleukin-1 antagonist rilonacept in Schnitzler syndrome: an open-label study. Allergy. 2012;67(7):943-950.

98. Kuemmerle-Deschner JB, et al. Canakinumab (ACZ885, a fully human IgG1 anti-IL-1beta mAb) induces sustained remission in pediatric patients with cryopyrin-associated periodic syndrome (CAPS). Arthritis Res Ther. 2011;13(1):R34.

99. Cavelti-Weder C, et al. Effects of gevokizumab on glycemia and inflammatory markers in type 2 diabetes. Diabetes Care. 2012;35(8):1654-1662.

100.Kuemmerle-Deschner JB, et al. Two-year results from an open-label, multicentre, phase III study evaluating the safety and efficacy of canakinumab in patients with cryopyrin-associated periodic syndrome across different severity phenotypes. Ann Rheum Dis. 2011;70(12):2095-2102.

101.Lachmann HJ, et al. Use of canakinumab in the cryopyrin-associated periodic syndrome. $\mathrm{NEnglJ}$ Med. 2009;360(23):2416-2425.

102. Schlesinger N, et al. Canakinumab for acute gouty arthritis in patients with limited treatment options: results from two randomised, multicentre, active-controlled, double-blind trials and their initial extensions. Ann Rheum Dis. 2012;71(11):1839-1848.

103. Moran A, et al. Interleukin-1 antagonism in type 1 diabetes of recent onset: two multicentre, randomised, double-blind, placebo-controlled trials. Lancet. 2013;381(9881):1905-1915.

104. Ridker PM, et al. Effects of interleukin-1beta inhibition with canakinumab on hemoglobin A1c, lipids, C-reactive protein, interleukin-6, and fibrinogen: a phase IIb randomized, placebo-controlled trial. Circulation. 2012;126(23):2739-2748.

105. Hensen J, Howard CP, Walter V, Thuren T. Impact of interleukin-1beta antibody (canakinumab) on glycaemic indicators in patients with type 2 diabetes mellitus: results of secondary endpoints from a randomized, placebo-controlled trial. Diabetes Metab. 2013;39(6):524-531.

106.Alten R, et al. Efficacy and safety of the human anti-IL-1beta monoclonal antibody canakinumab in rheumatoid arthritis: results of a 12-week, Phase II, dose-finding study. BMC Musculoskelet Disord. 2011;12:153.

107. Ruperto N, et al. Two randomized trials of canakinumab in systemic juvenile idiopathic arthritis. NEngl JMed. 2012;367(25):2396-2406.

108. de Koning HD, Schalkwijk J, van der VenJongekriig J, Stoffels M, van der Meer JW, Simon A. Sustained efficacy of the monoclonal antiinterleukin-1 beta antibody canakinumab in a 9-month trial in Schnitzler's syndrome. Ann Rheum Dis. 2013;72(10):1634-1638.

109. Ugurlu S, Ucar D, Seyahi E, Hatemi G, Yurdakul S. Canakinumab in a patient with juvenile Behcet's syndrome with refractory eye disease. Ann Rheum Dis. 2012;71(9):1589-1591.

110. Issafras H, Corbin JA, Goldfine ID, Roell MK. Detailed mechanistic analysis of gevokizumab, an allosteric anti-IL-1 $\beta$ antibody with differential receptor-modulating properties. J Pharmacol Exp Ther. 2014;348(1):202-215.

111. Roell MK, et al. Kinetic approach to pathway attenuation using XOMA 052, a regulatory therapeutic antibody that modulates interleukin-1beta activity.J Biol Chem. 2010;285(27):20607-20614.

112. Cavelti-Weder C, et al. Inhibition of IL-1 improves fatigue in type 2 diabetes. Diabetes Care. 2011;34(10):e158.

113. Gul A, et al. Interleukin-1ß-regulating antibody XOMA 052 (gevokizumab) in the treatment of acute exacerbations of resistant uveitis of Behcet's disease: an open-label pilot study. Ann Rheum Dis. 2012;71(4):563-566. 